\title{
Residue analysis and risk assessment of oxathiapiprolin and its metabolites in cucumber under field conditions
}

Wenzhuo Wang ${ }^{\dagger}$, Peipei Teng ${ }^{\dagger}$, Fengmao Liu ${ }^{\dagger *}$, Tingting Fan ${ }^{\dagger}$, Qingrong Peng ${ }^{\dagger}$, Zongyi Wang $\$$, Tongyao Hou

${ }^{\dagger}$ Department of Applied Chemistry, College of Science, China Agricultural University, Beijing 100193, People's Republic of China.

$\$$ Beijing Key Laboratory of Detection and Control of Spoilage Microorganisms and Pesticide Residues in Agricultural Products, Beijing University of Agriculture, Beijing 102206, People's Republic of China.

*Corresponding author:

Prof. Fengmao Liu, Pesticide Residue and Environmental Toxicology Lab, Department of Applied Chemistry, College of Science, China Agricultural University, No. 2 Yuanmingyuan West Road, Haidian District, Beijing, 100193 China.

Tel.: +861062731978

Fax: +861062733620

E-mail address: liufengmao@cau.edu.cn (Fengmao Liu*) 


\section{Support information}

Figure S1. Effect of purify agent amount on the recoveries of oxathiapiprolin parent and metabolites from the cucumber samples at the $0.2 \mathrm{mg} \mathrm{kg}^{-1}(\mathrm{n}=3)$.

Figure S2. Effect of the participation of anhydrous $\mathrm{MgSO}_{4}$ on the recoveries of oxathiapiprolin parent and metabolites from the cucumber samples at the $0.2 \mathrm{mg} \mathrm{kg}^{-1}$ $(n=3)$.

Figure S3. HPLC-MS/MS chromatograms of oxathiapiprolin and its two metabolites (IN-E8S72 and IN-SXS67) (a) in blank cucumber, and (b) fortified cucumber sample at $0.2 \mathrm{mg} \mathrm{kg}^{-1}$.

Table S1. Terminal residues of oxathiapiprolin and its metabolites in cucumber.

Table S2. Dissipation of oxathiapiprolin and its metabolites in cucumber.

Table S3. The IEDI for oxathiapiprolin. 


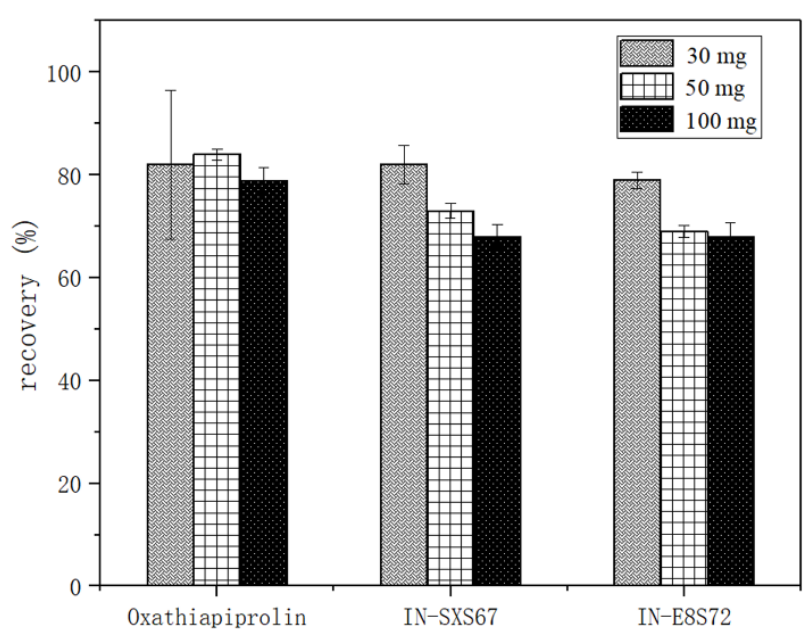

Figure S1. Effect of purify agent amount on the recoveries of oxathiapiprolin parent and metabolites from the cucumber samples at the $0.2 \mathrm{mg} \mathrm{kg}^{-1}(\mathrm{n}=3)$.

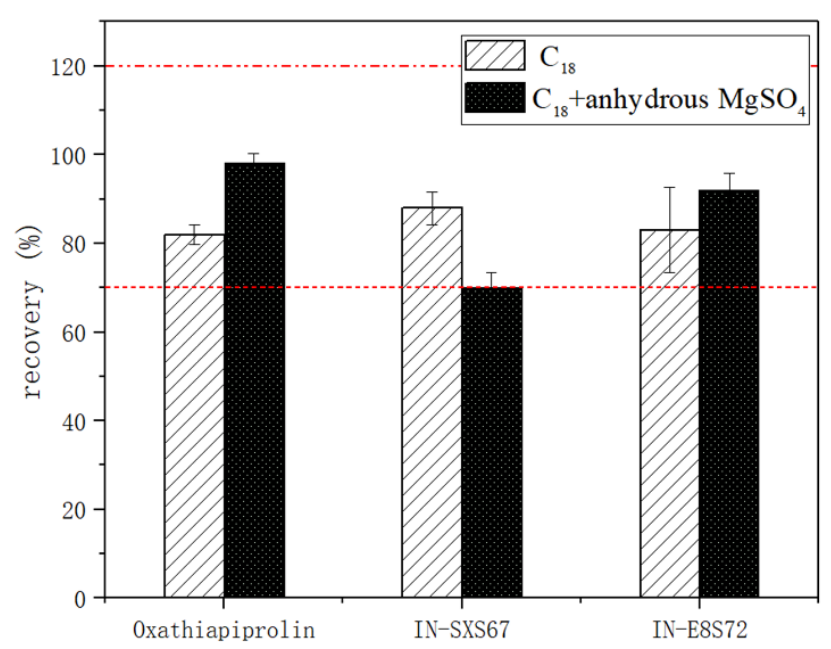

Figure S2. Effect of the participation of anhydrous $\mathrm{MgSO}_{4}$ on the recoveries of oxathiapiprolin parent and metabolites from the cucumber samples at the $0.2 \mathrm{mg} \mathrm{kg}^{-1}$ $(n=3)$. 


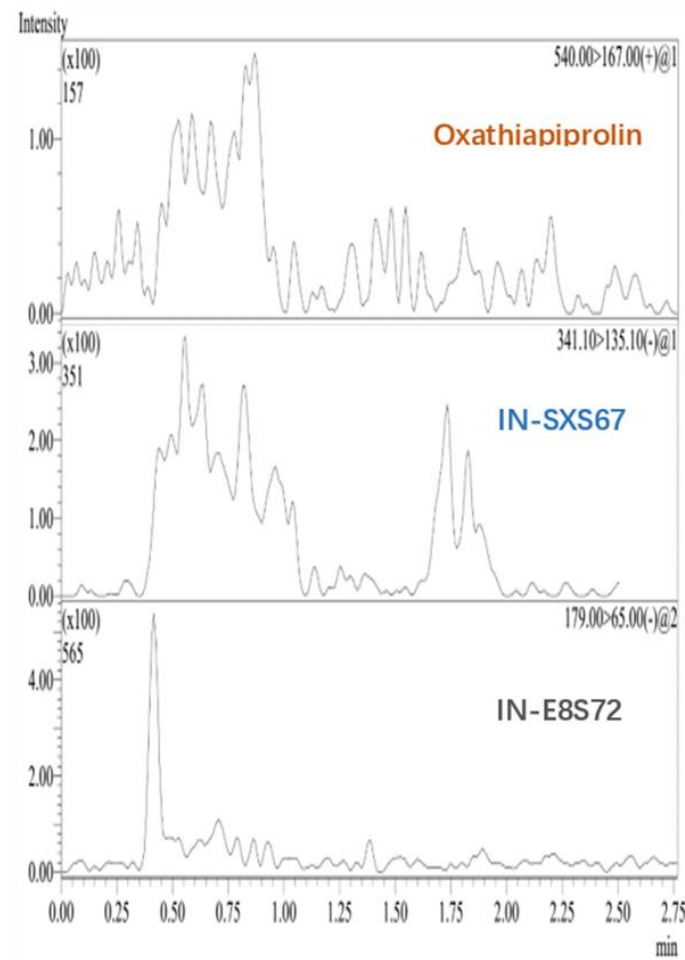

a)

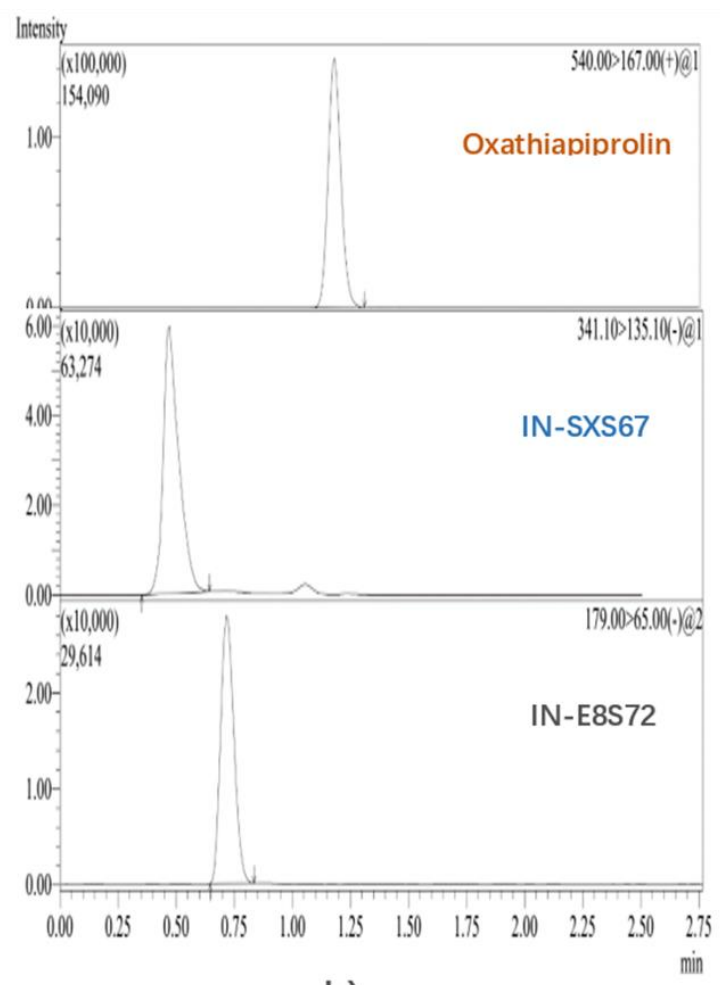

b)

Figure S3. HPLC-MS/MS chromatograms of oxathiapiprolin and its two metabolites (IN-E8S72 and IN-SXS67) (a) in blank cucumber, and (b) fortified cucumber sample at $0.2 \mathrm{mg} \mathrm{kg}^{-1}$. 
Table S1. Terminal residues of oxathiapiprolin and its metabolites in cucumber.

\begin{tabular}{|c|c|c|c|c|c|c|c|}
\hline \multirow{2}{*}{$\begin{array}{l}\text { Application rate }(\mathrm{g} \\
\left.\text { a.i. } \mathrm{ha}^{-1}\right)\end{array}$} & \multirow[t]{2}{*}{ Year } & \multirow[t]{2}{*}{ Trial site } & \multirow{2}{*}{$\begin{array}{l}\text { Cultivation } \\
\text { facilities }\end{array}$} & \multirow{2}{*}{$\begin{array}{l}\text { Preharvest } \\
\text { interval (PHI) }\end{array}$} & \multicolumn{3}{|c|}{ Residue $\left(\mathrm{mg} \mathrm{kg}^{-1}\right)$} \\
\hline & & & & & oxathiapiprolin & IN-E8S72 & IN-SXS67 \\
\hline \multirow[t]{21}{*}{16.875} & \multirow[t]{6}{*}{2017} & \multirow[t]{2}{*}{ Beijing } & \multirow[t]{2}{*}{ open field } & 3 & $<0.02$ & $<0.02$ & $<0.02$ \\
\hline & & & & 5 & $<0.02$ & $<0.02$ & $<0.02$ \\
\hline & & \multirow[t]{2}{*}{ Anhui } & \multirow[t]{2}{*}{ open field } & 3 & $<0.02$ & $<0.02$ & $<0.02$ \\
\hline & & & & 5 & $<0.02$ & $<0.02$ & $<0.02$ \\
\hline & & \multirow[t]{2}{*}{ Heilongjiang } & \multirow[t]{2}{*}{ greenhouse } & 3 & $<0.02$ & $<0.02$ & $<0.02$ \\
\hline & & & & 5 & $<0.02$ & $<0.02$ & $<0.02$ \\
\hline & \multirow[t]{15}{*}{2018} & \multirow[t]{2}{*}{ Beijing } & \multirow[t]{2}{*}{ greenhouse } & 3 & $<0.02$ & $<0.02$ & $<0.02$ \\
\hline & & & & 5 & $<0.02$ & $<0.02$ & $<0.02$ \\
\hline & & \multirow[t]{2}{*}{ Anhui } & \multirow[t]{2}{*}{ greenhouse } & 3 & $<0.02$ & $<0.02$ & $<0.02$ \\
\hline & & & & 5 & $<0.02$ & $<0.02$ & $<0.02$ \\
\hline & & \multirow[t]{2}{*}{ Henan } & \multirow{2}{*}{ greenhouse } & 3 & $<0.02$ & $<0.02$ & $<0.02$ \\
\hline & & & & 5 & $<0.02$ & $<0.02$ & $<0.02$ \\
\hline & & \multirow{2}{*}{ Shandong } & \multirow{2}{*}{ greenhouse } & 3 & $<0.02$ & $<0.02$ & $<0.02$ \\
\hline & & & & 5 & $<0.02$ & $<0.02$ & $<0.02$ \\
\hline & & \multirow[t]{2}{*}{ Shandong } & \multirow[t]{2}{*}{ open field } & 3 & $<0.02$ & $<0.02$ & $<0.02$ \\
\hline & & & & 5 & $<0.02$ & $<0.02$ & $<0.02$ \\
\hline & & \multirow[t]{2}{*}{ Hubei } & \multirow[t]{2}{*}{ open field } & 3 & $<0.02$ & $<0.02$ & $<0.02$ \\
\hline & & & & 5 & $<0.02$ & $<0.02$ & $<0.02$ \\
\hline & & \multirow[t]{2}{*}{ Ningxia } & \multirow[t]{2}{*}{ open field } & 3 & $<0.02$ & $<0.02$ & $<0.02$ \\
\hline & & & & 5 & $<0.02$ & $<0.02$ & $<0.02$ \\
\hline & & Sichuan & open field & 3 & $<0.02$ & $<0.02$ & $<0.02$ \\
\hline
\end{tabular}




\begin{tabular}{|c|c|c|c|c|c|c|c|}
\hline \multirow{5}{*}{$\begin{array}{l}\text { Application rate (g } \\
\left.\text { a.i. ha }{ }^{-1}\right)\end{array}$} & \multirow[t]{5}{*}{ Year } & \multirow[t]{3}{*}{ Trial site } & \multirow{3}{*}{$\begin{array}{l}\text { Cultivation } \\
\text { facilities }\end{array}$} & \multirow{2}{*}{$\begin{array}{l}\text { Preharvest } \\
\text { interval (PHI) }\end{array}$} & \multicolumn{3}{|c|}{ Residue (mg kg-1) } \\
\hline & & & & & oxathiapiprolin & IN-E8S72 & IN-SXS67 \\
\hline & & & & 5 & $<0.02$ & $<0.02$ & $<0.02$ \\
\hline & & \multirow[t]{2}{*}{ Heilongjiang } & \multirow[t]{2}{*}{ greenhouse } & 3 & $<0.02$ & $<0.02$ & $<0.02$ \\
\hline & & & & 5 & $<0.02$ & $<0.02$ & $<0.02$ \\
\hline
\end{tabular}

Table S2. Dissipation of oxathiapiprolin and its metabolites in cucumber.

\begin{tabular}{|c|c|c|c|c|}
\hline \multirow{2}{*}{ Sites } & \multirow{2}{*}{ Time after treatment (days) } & \multicolumn{3}{|c|}{ Residue (mg kg-1) } \\
\hline & & oxathiapiprolin & IN-E8S72 & IN-SXS67 \\
\hline \multirow{9}{*}{ Beijing } & 0.08 & 0.24 & $<0.02$ & $<0.02$ \\
\hline & 0.33 & 0.21 & $<0.02$ & $<0.02$ \\
\hline & 1 & 0.17 & $<0.02$ & $<0.02$ \\
\hline & 2 & 0.11 & $<0.02$ & $<0.02$ \\
\hline & 3 & 0.12 & $<0.02$ & $<0.02$ \\
\hline & 5 & 0.04 & $<0.02$ & $<0.02$ \\
\hline & 7 & 0.02 & $<0.02$ & $<0.02$ \\
\hline & 10 & $<0.02$ & $<0.02$ & $<0.02$ \\
\hline & 14 & $<0.02$ & $<0.02$ & $<0.02$ \\
\hline \multirow{4}{*}{ Anhui } & 0.08 & 0.037 & $<0.02$ & $<0.02$ \\
\hline & 0.33 & 0.026 & $<0.02$ & $<0.02$ \\
\hline & 1 & 0.023 & $<0.02$ & $<0.02$ \\
\hline & 2 & 0.02 & $<0.02$ & $<0.02$ \\
\hline
\end{tabular}




\begin{tabular}{|c|c|c|c|c|}
\hline \multirow{7}{*}{ Sites } & \multirow{2}{*}{ Time after treatment (days) } & \multicolumn{3}{|c|}{ Residue (mg kg-1) } \\
\hline & & oxathiapiprolin & IN-E8S72 & IN-SXS67 \\
\hline & 3 & 0.021 & $<0.02$ & $<0.02$ \\
\hline & 5 & 0.02 & $<0.02$ & $<0.02$ \\
\hline & 7 & $<0.02$ & $<0.02$ & $<0.02$ \\
\hline & 10 & $<0.02$ & $<0.02$ & $<0.02$ \\
\hline & 14 & $<0.02$ & $<0.02$ & $<0.02$ \\
\hline \multirow{9}{*}{ Heilongjiang } & 0.08 & 0.043 & $<0.02$ & $<0.02$ \\
\hline & 0.33 & 0.041 & $<0.02$ & $<0.02$ \\
\hline & 1 & 0.022 & $<0.02$ & $<0.02$ \\
\hline & 2 & 0.046 & $<0.02$ & $<0.02$ \\
\hline & 3 & 0.036 & $<0.02$ & $<0.02$ \\
\hline & 5 & 0.055 & $<0.02$ & $<0.02$ \\
\hline & 7 & $<0.02$ & $<0.02$ & $<0.02$ \\
\hline & 10 & $<0.02$ & $<0.02$ & $<0.02$ \\
\hline & 14 & $<0.02$ & $<0.02$ & $<0.02$ \\
\hline
\end{tabular}


Table S3. The IEDI for oxathiapiprolin

\begin{tabular}{|c|c|c|c|c|c|c|c|c|c|c|c|c|c|c|c|c|}
\hline $\begin{array}{l}\text { Codex } \\
\text { Code }\end{array}$ & Commodity description & $\begin{array}{r}\text { STMR } \\
\text { or } \\
\text { STMR-P } \\
\text { mg/kg }\end{array}$ & $\begin{array}{r}\text { G01 } \\
\text { (diet) }\end{array}$ & $\begin{array}{r}\text { G01 } \\
\text { intake }\end{array}$ & $\begin{array}{r}\text { G02 } \\
\text { (diet) }\end{array}$ & $\begin{array}{r}\text { G02 } \\
\text { intake }\end{array}$ & $\begin{array}{r}\text { G03 } \\
\text { (diet) }\end{array}$ & $\begin{array}{r}\text { G03 } \\
\text { intake }\end{array}$ & $\begin{array}{r}\text { G04 } \\
\text { (diet) }\end{array}$ & $\begin{array}{r}\text { G04 } \\
\text { intake }\end{array}$ & $\begin{array}{r}\text { G05 } \\
\text { (diet) }\end{array}$ & $\begin{array}{r}\text { G05 } \\
\text { intake }\end{array}$ & $\begin{array}{r}\text { G06 } \\
\text { (diet) }\end{array}$ & $\begin{array}{r}\text { G06 } \\
\text { intake }\end{array}$ & $\begin{array}{r}\text { G07 } \\
\text { (diet) }\end{array}$ & $\begin{array}{r}\text { G07 } \\
\text { intake }\end{array}$ \\
\hline $\begin{array}{l}\mathrm{FB} \\
2008\end{array}$ & $\begin{array}{l}\text { Small fruit vine climbing, raw } \\
\text { (incl processed) (i.e. grapes) }\end{array}$ & 0.21 & 16.25 & 3.41 & 28.96 & 6.08 & 2.87 & 0.60 & 24.22 & 5.09 & 9.33 & 1.96 & 68.64 & 14.41 & 142.23 & 29.87 \\
\hline $\begin{array}{l}\text { FB } \\
0269\end{array}$ & $\begin{array}{l}\text { Grape, raw (incl must, incl } \\
\text { dried, incl juice, incl wine) }\end{array}$ & 0.21 & 16.25 & 3.41 & 28.96 & 6.08 & 2.87 & 0.60 & 24.22 & 5.09 & 9.33 & 1.96 & 68.64 & 14.41 & 142.23 & 29.87 \\
\hline $\begin{array}{l}\text { FB } \\
0269\end{array}$ & $\begin{array}{l}\text { Grape, raw (incl must, incl } \\
\text { dried, incl wine, excl juice) }\end{array}$ & 0.21 & 16.07 & 3.37 & 28.60 & 6.01 & 2.81 & 0.59 & 23.85 & 5.01 & 9.03 & 1.90 & 68.58 & 14.40 & 141.53 & 29.72 \\
\hline $\begin{array}{l}\text { FB } \\
0269\end{array}$ & $\begin{array}{l}\text { Grape, raw (incl must, incl } \\
\text { juice, incl wine, excl dried) }\end{array}$ & 0.21 & 14.11 & 2.96 & 26.83 & 5.63 & 2.85 & 0.60 & 18.95 & 3.98 & 8.84 & 1.86 & 60.01 & 12.60 & 129.34 & 27.16 \\
\hline $\begin{array}{l}\text { FB } \\
0269\end{array}$ & $\begin{array}{l}\text { Grape, raw (incl dried, incl } \\
\text { juice, incl wine, excl must) }\end{array}$ & 0.21 & 15.91 & 3.34 & 28.84 & 6.06 & 2.87 & 0.60 & 24.20 & 5.08 & 9.33 & 1.96 & 68.62 & 14.41 & 142.07 & 29.83 \\
\hline $\begin{array}{l}\text { FB } \\
0269\end{array}$ & $\begin{array}{l}\text { Grape, raw (incl must, incl } \\
\text { dried, incl juice, excl wine) }\end{array}$ & 0.21 & 15.33 & 3.22 & 11.75 & 2.47 & 0.11 & 0.02 & 22.55 & 4.74 & 4.49 & 0.94 & 63.13 & 13.26 & 20.07 & 4.21 \\
\hline $\begin{array}{l}\text { FB } \\
0269\end{array}$ & $\begin{array}{l}\text { Grape, raw (incl must, incl } \\
\text { dried, excl juice, excl wine) }\end{array}$ & 0.21 & 15.15 & 3.18 & 11.38 & 2.39 & 0.10 & 0.02 & 22.18 & 4.66 & 4.19 & 0.88 & 63.07 & 13.24 & 19.37 & 4.07 \\
\hline $\begin{array}{l}\text { FB } \\
0269\end{array}$ & $\begin{array}{l}\text { Grape, raw (incl must, incl } \\
\text { juice, excl dried, excl wine) }\end{array}$ & 0.21 & 13.19 & 2.77 & 9.61 & 2.02 & 0.10 & 0.02 & 17.28 & 3.63 & 4.00 & 0.84 & 54.50 & 11.45 & 7.18 & 1.51 \\
\hline $\begin{array}{l}\text { FB } \\
0269\end{array}$ & $\begin{array}{l}\text { Grape, raw (incl must, incl } \\
\text { wine, excl dried, excl juice) }\end{array}$ & 0.21 & 13.94 & 2.93 & 26.46 & 5.56 & 2.79 & 0.59 & 18.58 & 3.90 & 8.54 & 1.79 & 59.95 & 12.59 & 128.64 & 27.01 \\
\hline
\end{tabular}




\begin{tabular}{|c|c|c|c|c|c|c|c|c|c|c|c|c|c|c|c|c|}
\hline $\begin{array}{l}\text { Codex } \\
\text { Code }\end{array}$ & Commodity description & $\begin{array}{r}\text { STMR } \\
\text { or } \\
\text { STMR-P } \\
\mathrm{mg} / \mathrm{kg}\end{array}$ & $\begin{array}{r}\text { G01 } \\
\text { (diet) }\end{array}$ & $\begin{array}{r}\text { G01 } \\
\text { intake }\end{array}$ & $\begin{array}{r}\text { G02 } \\
\text { (diet) }\end{array}$ & $\begin{array}{r}\text { G02 } \\
\text { intake }\end{array}$ & $\begin{array}{r}\text { G03 } \\
\text { (diet) }\end{array}$ & $\begin{array}{r}\text { G03 } \\
\text { intake }\end{array}$ & $\begin{array}{r}\text { G04 } \\
\text { (diet) }\end{array}$ & $\begin{array}{r}\text { G04 } \\
\text { intake }\end{array}$ & $\begin{array}{r}\text { G05 } \\
\text { (diet) }\end{array}$ & $\begin{array}{r}\text { G05 } \\
\text { intake }\end{array}$ & $\begin{array}{r}\text { G06 } \\
\text { (diet) }\end{array}$ & $\begin{array}{r}\text { G06 } \\
\text { intake }\end{array}$ & $\begin{array}{r}\text { G07 } \\
\text { (diet) }\end{array}$ & $\begin{array}{r}\text { G07 } \\
\text { intake }\end{array}$ \\
\hline $\begin{array}{l}\text { FB } \\
0269\end{array}$ & $\begin{array}{l}\text { Grape, raw (incl dried, incl } \\
\text { juice, excl wine, excl must) }\end{array}$ & 0.21 & 14.99 & 3.15 & 11.62 & 2.44 & 0.11 & 0.02 & 22.53 & 4.73 & 4.48 & 0.94 & 63.11 & 13.25 & 19.92 & 4.18 \\
\hline $\begin{array}{l}\text { FB } \\
0269\end{array}$ & $\begin{array}{l}\text { Grape, raw (incl dried, incl } \\
\text { wine, excl must, excl juice) }\end{array}$ & 0.21 & 15.74 & 3.31 & 28.47 & 5.98 & 2.81 & 0.59 & 23.83 & 5.00 & 9.03 & 1.90 & 68.56 & 14.40 & 141.38 & 29.69 \\
\hline $\begin{array}{l}\text { FB } \\
0269\end{array}$ & $\begin{array}{l}\text { Grape, raw (incl juice, incl } \\
\text { wine, excl must, excl dried) }\end{array}$ & 0.21 & 13.78 & 2.89 & 26.70 & 5.61 & 2.85 & 0.60 & 18.92 & 3.97 & 8.84 & 1.86 & 59.99 & 12.60 & 129.18 & 27.13 \\
\hline $\begin{array}{l}\text { FB } \\
0269\end{array}$ & $\begin{array}{l}\text { Grape, raw (incl must, excl } \\
\text { dried, excl juice, excl wine) }\end{array}$ & 0.21 & 13.02 & 2.73 & 9.25 & 1.94 & 0.10 & 0.02 & 16.91 & 3.55 & 3.70 & 0.78 & 54.44 & 11.43 & 6.48 & 1.36 \\
\hline $\begin{array}{l}\text { FB } \\
0269\end{array}$ & $\begin{array}{l}\text { Grape, raw (incl dried, excl } \\
\text { must, excl juice, excl wine) }\end{array}$ & 0.21 & 14.82 & 3.11 & 11.26 & 2.36 & 0.10 & 0.02 & 22.16 & 4.65 & 4.19 & 0.88 & 63.05 & 13.24 & 19.22 & 4.04 \\
\hline $\begin{array}{l}\text { FB } \\
0269\end{array}$ & $\begin{array}{l}\text { Grape, raw (incl juice, excl } \\
\text { must, excl dried, excl wine) }\end{array}$ & 0.21 & 12.86 & 2.70 & 9.49 & 1.99 & 0.10 & 0.02 & 17.25 & 3.62 & 3.99 & 0.84 & 54.48 & 11.44 & 7.03 & 1.48 \\
\hline $\begin{array}{l}\text { FB } \\
0269\end{array}$ & $\begin{array}{l}\text { Grape, raw (incl wine, excl } \\
\text { must, excl dried, excl juice) }\end{array}$ & 0.21 & 13.60 & 2.86 & 26.34 & 5.53 & 2.79 & 0.59 & 18.55 & 3.90 & 8.54 & 1.79 & 59.93 & 12.59 & 128.49 & 26.98 \\
\hline $\begin{array}{l}\text { FB } \\
0269\end{array}$ & Grape, raw & 0.21 & 12.68 & 2.66 & 9.12 & 1.92 & 0.10 & 0.02 & 16.88 & 3.54 & 3.70 & 0.78 & 54.42 & 11.43 & 6.33 & 1.33 \\
\hline- & Grape must & 0.13 & 0.33 & 0.04 & 0.13 & 0.02 & 0.10 & 0.01 & 0.10 & 0.01 & 0.10 & 0.01 & 0.10 & 0.01 & 0.16 & 0.02 \\
\hline $\begin{array}{l}\mathrm{DF} \\
0269\end{array}$ & $\begin{array}{l}\text { Grape, dried (= currants, } \\
\text { raisins and sultanas) }\end{array}$ & 0.29 & 0.51 & 0.15 & 0.51 & 0.15 & 0.10 & 0.03 & 1.27 & 0.37 & 0.12 & 0.03 & 2.07 & 0.60 & 3.09 & 0.90 \\
\hline JF 0269 & Grape juice & 0.034 & 0.14 & 0.00 & 0.29 & 0.01 & 0.10 & 0.00 & 0.30 & 0.01 & 0.24 & 0.01 & 0.10 & 0.00 & 0.56 & 0.02 \\
\hline- & Grape wine (incl vermouths) & 0.029 & 0.67 & 0.02 & 12.53 & 0.36 & 2.01 & 0.06 & 1.21 & 0.04 & 3.53 & 0.10 & 4.01 & 0.12 & 88.93 & 2.58 \\
\hline - & Onions, mature bulbs, dry & 0.01 & 29.36 & 0.29 & 37.50 & 0.38 & 3.56 & 0.04 & 34.78 & 0.35 & 18.81 & 0.19 & 43.38 & 0.43 & 19.69 & 0.20 \\
\hline
\end{tabular}




\begin{tabular}{|c|c|c|c|c|c|c|c|c|c|c|c|c|c|c|c|c|}
\hline $\begin{array}{l}\text { Codex } \\
\text { Code }\end{array}$ & Commodity description & $\begin{array}{r}\text { STMR } \\
\text { or } \\
\text { STMR-P } \\
\mathrm{mg} / \mathrm{kg}\end{array}$ & $\begin{array}{r}\text { G01 } \\
\text { (diet) }\end{array}$ & $\begin{array}{r}\text { G01 } \\
\text { intake }\end{array}$ & $\begin{array}{r}\text { G02 } \\
\text { (diet) }\end{array}$ & $\begin{array}{r}\text { G02 } \\
\text { intake }\end{array}$ & $\begin{array}{r}\text { G03 } \\
\text { (diet) }\end{array}$ & $\begin{array}{r}\text { G03 } \\
\text { intake }\end{array}$ & $\begin{array}{r}\text { G04 } \\
\text { (diet) }\end{array}$ & $\begin{array}{r}\text { G04 } \\
\text { intake }\end{array}$ & $\begin{array}{r}\text { G05 } \\
\text { (diet) }\end{array}$ & $\begin{array}{r}\text { G05 } \\
\text { intake }\end{array}$ & $\begin{array}{r}\text { G06 } \\
\text { (diet) }\end{array}$ & $\begin{array}{r}\text { G06 } \\
\text { intake }\end{array}$ & $\begin{array}{r}\text { G07 } \\
\text { (diet) }\end{array}$ & $\begin{array}{r}\text { G07 } \\
\text { intake }\end{array}$ \\
\hline- & Onions, green, raw & 0.6 & 2.45 & 1.47 & 1.49 & 0.89 & 1.02 & 0.61 & 2.60 & 1.56 & 0.60 & 0.36 & 2.03 & 1.22 & 1.55 & 0.93 \\
\hline $\begin{array}{l}\text { VB } \\
0041\end{array}$ & Cabbages, head, raw & 0.14 & 2.73 & 0.38 & 27.92 & 3.91 & 0.55 & 0.08 & 4.47 & 0.63 & 4.27 & 0.60 & 10.25 & 1.44 & 8.97 & 1.26 \\
\hline $\begin{array}{l}\text { VB } \\
0400\end{array}$ & Broccoli, raw & 0.22 & 0.88 & 0.19 & 0.17 & 0.04 & 0.10 & 0.02 & 1.25 & 0.28 & 3.00 & 0.66 & 1.09 & 0.24 & 4.24 & 0.93 \\
\hline $\begin{array}{l}\text { VB } \\
0404\end{array}$ & Cauliflower, raw & 0.08 & 1.65 & 0.13 & 0.32 & 0.03 & 0.10 & 0.01 & 2.33 & 0.19 & 4.79 & 0.38 & 2.03 & 0.16 & 5.27 & 0.42 \\
\hline $\begin{array}{l}\mathrm{VC} \\
0046\end{array}$ & $\begin{array}{l}\text { Melons, raw (excl } \\
\text { watermelons) }\end{array}$ & 0.03 & 8.90 & 0.27 & 8.64 & 0.26 & 0.80 & 0.02 & 17.90 & 0.54 & 2.80 & 0.08 & 29.17 & 0.88 & 9.20 & 0.28 \\
\hline $\begin{array}{l}\text { VC } \\
0424\end{array}$ & Cucumber, raw & 0.06 & 8.01 & 0.48 & 30.66 & 1.84 & 1.45 & 0.09 & 19.84 & 1.19 & 0.27 & 0.02 & 34.92 & 2.10 & 6.72 & 0.40 \\
\hline $\begin{array}{l}\text { VO } \\
0448\end{array}$ & $\begin{array}{l}\text { Tomato, raw (incl juice, incl } \\
\text { paste, incl canned) }\end{array}$ & 0.04 & 51.75 & 2.07 & 81.80 & 3.27 & 16.99 & 0.68 & 102.02 & 4.08 & 26.32 & 1.05 & 214.77 & 8.59 & 64.74 & 2.59 \\
\hline $\begin{array}{l}\text { VO } \\
0448\end{array}$ & $\begin{array}{l}\text { Tomato, raw (incl juice, incl } \\
\text { paste, excl canned) }\end{array}$ & 0.04 & 51.44 & 2.06 & 81.32 & 3.25 & 16.96 & 0.68 & 100.30 & 4.01 & 26.16 & 1.05 & 212.43 & 8.50 & 52.99 & 2.12 \\
\hline $\begin{array}{l}\text { VO } \\
0448\end{array}$ & $\begin{array}{l}\text { Tomato, raw (incl juice, incl } \\
\text { canned, excl paste) }\end{array}$ & 0.04 & 42.41 & 1.70 & 76.50 & 3.06 & 10.69 & 0.43 & 85.07 & 3.40 & 24.98 & 1.00 & 203.44 & 8.14 & 44.88 & 1.80 \\
\hline $\begin{array}{l}\text { VO } \\
0448\end{array}$ & $\begin{array}{l}\text { Tomato, raw (incl paste, incl } \\
\text { canned, excl juice) }\end{array}$ & 0.04 & 51.39 & 2.06 & 81.44 & 3.26 & 16.99 & 0.68 & 101.55 & 4.06 & 26.26 & 1.05 & 214.59 & 8.58 & 63.73 & 2.55 \\
\hline $\begin{array}{l}\text { VO } \\
0448\end{array}$ & $\begin{array}{l}\text { Tomato, raw (incl juice, excl } \\
\text { paste, excl canned) }\end{array}$ & 0.04 & 42.09 & 1.68 & 76.01 & 3.04 & 10.67 & 0.43 & 83.35 & 3.33 & 24.82 & 0.99 & 201.11 & 8.04 & 33.13 & 1.33 \\
\hline
\end{tabular}




\begin{tabular}{|c|c|c|c|c|c|c|c|c|c|c|c|c|c|c|c|c|}
\hline $\begin{array}{l}\text { Codex } \\
\text { Code }\end{array}$ & Commodity description & $\begin{array}{r}\text { STMR } \\
\text { or } \\
\text { STMR-P } \\
\mathrm{mg} / \mathrm{kg}\end{array}$ & $\begin{array}{r}\text { G01 } \\
\text { (diet) }\end{array}$ & $\begin{array}{r}\text { G01 } \\
\text { intake }\end{array}$ & $\begin{array}{r}\text { G02 } \\
\text { (diet) }\end{array}$ & $\begin{array}{r}\text { G02 } \\
\text { intake }\end{array}$ & $\begin{array}{r}\text { G03 } \\
\text { (diet) }\end{array}$ & $\begin{array}{r}\text { G03 } \\
\text { intake }\end{array}$ & $\begin{array}{r}\text { G04 } \\
\text { (diet) }\end{array}$ & $\begin{array}{r}\text { G04 } \\
\text { intake }\end{array}$ & $\begin{array}{r}\text { G05 } \\
\text { (diet) }\end{array}$ & $\begin{array}{r}\text { G05 } \\
\text { intake }\end{array}$ & $\begin{array}{r}\text { G06 } \\
\text { (diet) }\end{array}$ & $\begin{array}{r}\text { G06 } \\
\text { intake }\end{array}$ & $\begin{array}{r}\text { G07 } \\
\text { (diet) }\end{array}$ & $\begin{array}{r}\text { G07 } \\
\text { intake }\end{array}$ \\
\hline $\begin{array}{l}\text { VO } \\
0448\end{array}$ & $\begin{array}{l}\text { Tomato, raw (incl paste, excl } \\
\text { juice, excl canned) }\end{array}$ & 0.04 & 51.07 & 2.04 & 80.96 & 3.24 & 16.96 & 0.68 & 99.83 & 3.99 & 26.09 & 1.04 & 212.26 & 8.49 & 51.98 & 2.08 \\
\hline $\begin{array}{l}\text { VO } \\
0448\end{array}$ & $\begin{array}{l}\text { Tomato, raw (incl canned, } \\
\text { excl juice, excl paste) }\end{array}$ & 0.04 & 42.04 & 1.68 & 76.13 & 3.05 & 10.69 & 0.43 & 84.59 & 3.38 & 24.92 & 1.00 & 203.27 & 8.13 & 43.88 & 1.76 \\
\hline $\begin{array}{l}\text { VO } \\
0448\end{array}$ & Tomato, raw & 0.04 & 41.73 & 1.67 & 75.65 & 3.03 & 10.66 & 0.43 & 82.87 & 3.31 & 24.75 & 0.99 & 200.93 & 8.04 & 32.13 & 1.29 \\
\hline- & Tomato, canned (\& peeled) & 0.0016 & 0.20 & 0.00 & 0.31 & 0.00 & 0.10 & 0.00 & 1.11 & 0.00 & 0.11 & 0.00 & 1.50 & 0.00 & 7.57 & 0.01 \\
\hline- & $\begin{array}{l}\text { Tomato, paste (i.e. } \\
\text { concentrated tomato } \\
\text { sauce/puree) }\end{array}$ & 0.044 & 2.34 & 0.10 & 1.33 & 0.06 & 1.57 & 0.07 & 4.24 & 0.19 & 0.34 & 0.01 & 2.83 & 0.12 & 4.96 & 0.22 \\
\hline JF 0448 & $\begin{array}{l}\text { Tomato, juice (single strength, } \\
\text { incl concentrated) }\end{array}$ & 0.006 & 0.29 & 0.00 & 0.29 & 0.00 & 0.10 & 0.00 & 0.38 & 0.00 & 0.10 & 0.00 & 0.14 & 0.00 & 0.80 & 0.00 \\
\hline $\begin{array}{l}\mathrm{VL} \\
0502\end{array}$ & Spinach, raw & 3.7 & 0.74 & 2.74 & 0.22 & 0.81 & 0.10 & 0.37 & 0.91 & 3.37 & 0.10 & 0.37 & 2.92 & 10.80 & 2.20 & 8.14 \\
\hline $\begin{array}{l}\text { VP } \\
0064\end{array}$ & $\begin{array}{l}\text { Peas, green, without pods, raw } \\
\text { (i.e. immature seeds only) } \\
\text { (Pisum spp) }\end{array}$ & 0.09 & 1.97 & 0.18 & 0.51 & 0.05 & 0.10 & 0.01 & 0.79 & 0.07 & 3.68 & 0.33 & 3.80 & 0.34 & 10.72 & 0.96 \\
\hline \multicolumn{3}{|c|}{ Total intake (ug/person) } & \multicolumn{2}{|r|}{75.0} & \multicolumn{2}{|r|}{107} & \multicolumn{2}{|r|}{12.5} & \multicolumn{2}{|r|}{114.8} & \multicolumn{2}{|r|}{35.2} & \multicolumn{2}{|r|}{313.1} & \multicolumn{2}{|r|}{314.4} \\
\hline \multicolumn{3}{|c|}{ Bodyweight per region $(\mathrm{kg} \mathrm{bw})=$} & \multicolumn{2}{|r|}{60.0} & \multicolumn{2}{|r|}{60} & \multicolumn{2}{|r|}{60} & \multicolumn{2}{|r|}{60} & \multicolumn{2}{|r|}{60} & \multicolumn{2}{|r|}{60} & \multicolumn{2}{|r|}{60} \\
\hline \multicolumn{3}{|r|}{$\% \mathrm{ADI}=$} & \multicolumn{2}{|r|}{$0.03 \%$} & \multicolumn{2}{|r|}{$0.04 \%$} & \multicolumn{2}{|r|}{$0.01 \%$} & \multicolumn{2}{|r|}{$0.05 \%$} & \multicolumn{2}{|r|}{$0.01 \%$} & \multicolumn{2}{|r|}{$0.13 \%$} & \multicolumn{2}{|r|}{$0.13 \%$} \\
\hline
\end{tabular}




\begin{tabular}{|c|c|c|c|c|c|c|c|c|c|c|c|c|c|c|c|c|c|c|}
\hline $\begin{array}{l}\text { Codex } \\
\text { Code }\end{array}$ & Commodity description & $\begin{array}{r}\text { STMR or } \\
\text { STMR-P } \\
\mathrm{mg} / \mathrm{kg}\end{array}$ & $\begin{array}{r}\text { G08 } \\
\text { (diet) }\end{array}$ & $\begin{array}{r}\text { G08 } \\
\text { intake }\end{array}$ & $\begin{array}{r}\text { G09 } \\
\text { (diet) }\end{array}$ & $\begin{array}{r}\text { G09 } \\
\text { intake }\end{array}$ & $\begin{array}{r}\text { G10 } \\
\text { (diet) }\end{array}$ & $\begin{array}{r}\text { G10 } \\
\text { intake }\end{array}$ & $\begin{array}{r}\text { G11 } \\
\text { (diet) }\end{array}$ & $\begin{array}{r}\text { G11 } \\
\text { intake }\end{array}$ & $\begin{array}{r}\text { G12 } \\
\text { (diet) }\end{array}$ & $\begin{array}{r}\text { G12 } \\
\text { intake }\end{array}$ & $\begin{array}{r}\text { G13 } \\
\text { (diet) }\end{array}$ & $\begin{array}{r}\text { G13 } \\
\text { intake }\end{array}$ & $\begin{array}{r}\text { G14 } \\
\text { (diet) }\end{array}$ & $\begin{array}{r}\text { G14 } \\
\text { intak } \\
\text { e }\end{array}$ & $\begin{array}{r}\text { G15 } \\
\text { (diet) }\end{array}$ & $\begin{array}{r}\text { G15 } \\
\text { intake }\end{array}$ \\
\hline FB 2008 & $\begin{array}{l}\text { Small fruit vine climbing, } \\
\text { raw (incl processed) (i.e. } \\
\text { grapes) }\end{array}$ & 0.21 & 105.77 & 22.21 & 7.87 & 1.65 & 52.44 & 11.01 & $\begin{array}{r}109.2 \\
2\end{array}$ & 22.94 & 10.96 & 2.30 & 0.17 & 0.63 & 0.10 & 0.37 & 0.81 & 3.00 \\
\hline FB 0269 & $\begin{array}{l}\text { Grape, raw (incl must, incl } \\
\text { dried, incl juice, incl wine ) }\end{array}$ & 0.21 & 105.77 & 22.21 & 7.87 & 1.65 & 52.44 & 11.01 & $\begin{array}{r}109.2 \\
2\end{array}$ & 22.94 & 10.96 & 2.30 & 0.17 & 0.63 & 0.10 & 0.37 & 0.81 & 3.00 \\
\hline FB 0269 & $\begin{array}{l}\text { Grape, raw (incl must, incl } \\
\text { dried, incl wine, excl juice) }\end{array}$ & 0.21 & 103.35 & 21.70 & 7.85 & 1.65 & 49.67 & 10.43 & $\begin{array}{r}106.4 \\
2\end{array}$ & 22.35 & 10.53 & 2.21 & 0.17 & 0.63 & 0.10 & 0.37 & 0.81 & 3.00 \\
\hline FB 0269 & $\begin{array}{l}\text { Grape, raw (incl must, incl } \\
\text { juice, incl wine, excl dried) }\end{array}$ & 0.21 & 99.46 & 20.89 & 7.76 & 1.63 & 46.71 & 9.81 & 91.48 & 19.21 & 9.23 & 1.94 & 0.17 & 0.63 & 0.10 & 0.37 & 0.81 & 3.00 \\
\hline FB 0269 & $\begin{array}{l}\text { Grape, raw (incl dried, incl } \\
\text { juice, incl wine, excl must) }\end{array}$ & 0.21 & 105.68 & 22.19 & 7.87 & 1.65 & 52.32 & 10.99 & $\begin{array}{r}109.1 \\
1\end{array}$ & 22.91 & 10.96 & 2.30 & 0.17 & 0.63 & 0.10 & 0.37 & 0.81 & 3.00 \\
\hline FB 0269 & $\begin{array}{l}\text { Grape, raw (incl must, incl } \\
\text { dried, incl juice, excl wine) }\end{array}$ & 0.21 & 20.04 & 4.21 & 5.35 & 1.12 & 18.01 & 3.78 & 25.20 & 5.29 & 2.94 & 0.62 & 0.17 & 0.63 & 0.10 & 0.37 & 0.81 & 3.00 \\
\hline FB 0269 & $\begin{array}{l}\text { Grape, raw (incl must, incl } \\
\text { dried, excl juice, excl wine) }\end{array}$ & 0.21 & 17.62 & 3.70 & 5.33 & 1.12 & 15.24 & 3.20 & 22.40 & 4.70 & 2.51 & 0.53 & 0.17 & 0.63 & 0.10 & 0.37 & 0.81 & 3.00 \\
\hline FB 0269 & $\begin{array}{l}\text { Grape, raw (incl must, incl } \\
\text { juice, excl dried, excl wine) }\end{array}$ & 0.21 & 13.73 & 2.88 & 5.24 & 1.10 & 12.27 & 2.58 & 7.46 & 1.57 & 1.21 & 0.25 & 0.17 & 0.63 & 0.10 & 0.37 & 0.81 & 3.00 \\
\hline FB 0269 & $\begin{array}{l}\text { Grape, raw (incl must, incl } \\
\text { wine, excl dried, excl juice) }\end{array}$ & 0.21 & 97.04 & 20.38 & 7.74 & 1.63 & 43.94 & 9.23 & 88.68 & 18.62 & 8.80 & 1.85 & 0.17 & 0.63 & 0.10 & 0.37 & 0.81 & 3.00 \\
\hline FB 0269 & $\begin{array}{l}\text { Grape, raw (incl dried, incl } \\
\text { juice, excl wine, excl must) }\end{array}$ & 0.21 & 19.96 & 4.19 & 5.35 & 1.12 & 17.88 & 3.75 & 25.09 & 5.27 & 2.94 & 0.62 & 0.17 & 0.63 & 0.10 & 0.37 & 0.81 & 3.00 \\
\hline FB 0269 & $\begin{array}{l}\text { Grape, raw (incl dried, incl } \\
\text { wine, excl must, excl juice) }\end{array}$ & 0.21 & 103.26 & 21.68 & 7.84 & 1.65 & 49.55 & 10.41 & $\begin{array}{r}106.3 \\
1\end{array}$ & 22.33 & 10.53 & 2.21 & 0.17 & 0.63 & 0.10 & 0.37 & 0.81 & 3.00 \\
\hline
\end{tabular}




\begin{tabular}{|c|c|c|c|c|c|c|c|c|c|c|c|c|c|c|c|c|c|c|}
\hline $\begin{array}{l}\text { Codex } \\
\text { Code }\end{array}$ & Commodity description & $\begin{array}{r}\text { STMR or } \\
\text { STMR-P } \\
\mathrm{mg} / \mathrm{kg}\end{array}$ & $\begin{array}{r}\text { G08 } \\
\text { (diet) }\end{array}$ & $\begin{array}{r}\text { G08 } \\
\text { intake }\end{array}$ & $\begin{array}{r}\text { G09 } \\
\text { (diet) }\end{array}$ & $\begin{array}{r}\text { G09 } \\
\text { intake }\end{array}$ & $\begin{array}{r}\text { G10 } \\
\text { (diet) }\end{array}$ & $\begin{array}{r}\text { G10 } \\
\text { intake }\end{array}$ & $\begin{array}{r}\text { G11 } \\
\text { (diet) }\end{array}$ & $\begin{array}{r}\text { G11 } \\
\text { intake }\end{array}$ & $\begin{array}{r}\text { G12 } \\
\text { (diet) }\end{array}$ & $\begin{array}{r}\text { G12 } \\
\text { intake }\end{array}$ & $\begin{array}{r}\text { G13 } \\
\text { (diet) }\end{array}$ & $\begin{array}{r}\text { G13 } \\
\text { intake }\end{array}$ & $\begin{array}{r}\text { G14 } \\
\text { (diet) }\end{array}$ & $\begin{array}{r}\text { G14 } \\
\text { intak } \\
\text { e }\end{array}$ & $\begin{array}{r}\text { G15 } \\
\text { (diet) }\end{array}$ & $\begin{array}{r}\text { G15 } \\
\text { intake }\end{array}$ \\
\hline FB 0269 & $\begin{array}{l}\text { Grape, raw (incl juice, incl } \\
\text { wine, excl must, excl dried) }\end{array}$ & 0.21 & 99.38 & 20.87 & 7.75 & 1.63 & 46.58 & 9.78 & 91.37 & 19.19 & 9.23 & 1.94 & 0.17 & 0.63 & 0.10 & 0.37 & 0.81 & 3.00 \\
\hline FB 0269 & $\begin{array}{l}\text { Grape, raw (incl must, excl } \\
\text { dried, excl juice, excl wine) }\end{array}$ & 0.21 & 11.31 & 2.38 & 5.21 & 1.09 & 9.50 & 2.00 & 4.66 & 0.98 & 0.78 & 0.16 & 0.17 & 0.63 & 0.10 & 0.37 & 0.81 & 3.00 \\
\hline FB 0269 & $\begin{array}{l}\text { Grape, raw (incl dried, excl } \\
\text { must, excl juice, excl wine) }\end{array}$ & 0.21 & 17.53 & 3.68 & 5.32 & 1.12 & 15.12 & 3.18 & 22.29 & 4.68 & 2.51 & 0.53 & 0.17 & 0.63 & 0.10 & 0.37 & 0.81 & 3.00 \\
\hline FB 0269 & $\begin{array}{l}\text { Grape, raw (incl juice, excl } \\
\text { must, excl dried, excl wine) }\end{array}$ & 0.21 & 13.65 & 2.87 & 5.23 & 1.10 & 12.15 & 2.55 & 7.35 & 1.54 & 1.21 & 0.25 & 0.17 & 0.63 & 0.10 & 0.37 & 0.81 & 3.00 \\
\hline FB 0269 & $\begin{array}{l}\text { Grape, raw (incl wine, excl } \\
\text { must, excl dried, excl juice) }\end{array}$ & 0.21 & 96.95 & 20.36 & 7.73 & 1.62 & 43.82 & 9.20 & 88.57 & 18.60 & 8.80 & 1.85 & 0.17 & 0.63 & 0.10 & 0.37 & 0.81 & 3.00 \\
\hline FB 0269 & Grape, raw & 0.21 & 11.22 & 2.36 & 5.21 & 1.09 & 9.38 & 1.97 & 4.55 & 0.96 & 0.78 & 0.16 & 0.17 & 0.63 & 0.10 & 0.37 & 0.81 & 3.00 \\
\hline- & Grape must & 0.13 & 0.10 & 0.01 & 0.10 & 0.01 & 0.12 & 0.02 & 0.11 & 0.01 & $\mathrm{NC}$ & - & 0.17 & 0.63 & 0.10 & 0.37 & 0.81 & 3.00 \\
\hline DF 0269 & $\begin{array}{l}\text { Grape, dried (= currants, } \\
\text { raisins and sultanas) }\end{array}$ & 0.29 & 1.51 & 0.44 & 0.10 & 0.03 & 1.38 & 0.40 & 4.26 & 1.24 & 0.42 & 0.12 & 0.17 & 0.63 & 0.10 & 0.37 & 0.81 & 3.00 \\
\hline JF 0269 & Grape juice & 0.034 & 1.96 & 0.07 & 0.10 & 0.00 & 2.24 & 0.08 & 2.27 & 0.08 & 0.34 & 0.01 & 0.17 & 0.63 & 0.10 & 0.37 & 0.81 & 3.00 \\
\hline - & $\begin{array}{l}\text { Grape wine (incl } \\
\text { vermouths) }\end{array}$ & 0.029 & 62.41 & 1.81 & 1.84 & 0.05 & 25.07 & 0.73 & 61.17 & 1.77 & 5.84 & 0.17 & 0.17 & 0.63 & 0.10 & 0.37 & 0.81 & 3.00 \\
\hline- & Onions, mature bulbs, dry & 0.01 & 29.83 & 0.30 & 24.64 & 0.25 & 31.35 & 0.31 & 9.72 & 0.10 & 12.59 & 0.13 & 0.17 & 0.63 & 0.10 & 0.37 & 0.81 & 3.00 \\
\hline- & Onions, green, raw & 0.6 & 0.74 & 0.44 & 1.05 & 0.63 & 3.74 & 2.24 & 0.94 & 0.56 & 6.45 & 3.87 & 0.17 & 0.63 & 0.10 & 0.37 & 0.81 & 3.00 \\
\hline VB 0041 & Cabbages, head, raw & 0.14 & 27.12 & 3.80 & 1.44 & 0.20 & 24.96 & 3.49 & 4.55 & 0.64 & 11.23 & 1.57 & 0.17 & 0.63 & 0.10 & 0.37 & 0.81 & 3.00 \\
\hline VB 0400 & Broccoli, raw & 0.22 & 1.76 & 0.39 & $\mathrm{NC}$ & - & 0.51 & 0.11 & 3.79 & 0.83 & 0.26 & 0.06 & 0.17 & 0.63 & 0.10 & 0.37 & 0.81 & 3.00 \\
\hline VB 0404 & Cauliflower, raw & 0.08 & 5.01 & 0.40 & $\mathrm{NC}$ & - & 2.70 & 0.22 & 5.57 & 0.45 & 0.49 & 0.04 & 0.17 & 0.63 & 0.10 & 0.37 & 0.81 & 3.00 \\
\hline
\end{tabular}




\begin{tabular}{|c|c|c|c|c|c|c|c|c|c|c|c|c|c|c|c|c|c|c|}
\hline $\begin{array}{l}\text { Codex } \\
\text { Code }\end{array}$ & Commodity description & $\begin{array}{r}\text { STMR or } \\
\text { STMR-P } \\
\mathrm{mg} / \mathrm{kg}\end{array}$ & $\begin{array}{r}\text { G08 } \\
\text { (diet) }\end{array}$ & $\begin{array}{r}\text { G08 } \\
\text { intake }\end{array}$ & $\begin{array}{r}\text { G09 } \\
\text { (diet) }\end{array}$ & $\begin{array}{r}\text { G09 } \\
\text { intake }\end{array}$ & $\begin{array}{r}\text { G10 } \\
\text { (diet) }\end{array}$ & $\begin{array}{r}\text { G10 } \\
\text { intake }\end{array}$ & $\begin{array}{r}\text { G11 } \\
\text { (diet) }\end{array}$ & $\begin{array}{r}\text { G11 } \\
\text { intake }\end{array}$ & $\begin{array}{r}\text { G12 } \\
\text { (diet) }\end{array}$ & $\begin{array}{r}\text { G12 } \\
\text { intake }\end{array}$ & $\begin{array}{r}\text { G13 } \\
\text { (diet) }\end{array}$ & $\begin{array}{r}\text { G13 } \\
\text { intake }\end{array}$ & $\begin{array}{r}\text { G14 } \\
\text { (diet) }\end{array}$ & $\begin{array}{r}\text { G14 } \\
\text { intak } \\
\text { e }\end{array}$ & $\begin{array}{r}\text { G15 } \\
\text { (diet) }\end{array}$ & $\begin{array}{r}\text { G15 } \\
\text { intake }\end{array}$ \\
\hline VC 0046 & $\begin{array}{l}\text { Melons, raw (excl } \\
\text { watermelons) }\end{array}$ & 0.03 & 11.95 & 0.36 & 14.63 & 0.44 & 8.99 & 0.27 & 7.86 & 0.24 & 2.46 & 0.07 & 0.17 & 0.63 & 0.10 & 0.37 & 0.81 & 3.00 \\
\hline VC 0424 & Cucumber, raw & 0.06 & 11.03 & 0.66 & 32.10 & 1.93 & 15.10 & 0.91 & 4.05 & 0.24 & 9.57 & 0.57 & 0.17 & 0.63 & 0.10 & 0.37 & 0.81 & 3.00 \\
\hline VO 0444 & Peppers, chili, raw & 0.04 & 14.00 & 5.60 & 8.25 & 3.30 & 5.77 & 2.31 & 6.44 & 2.58 & & & 0.17 & 0.63 & 0.10 & 0.37 & 0.81 & 3.00 \\
\hline VO 0448 & $\begin{array}{l}\text { Tomato, raw (incl juice, } \\
\text { incl paste, incl canned) }\end{array}$ & 0.04 & 68.31 & 2.73 & 36.05 & 1.44 & 82.09 & 3.28 & 54.50 & 2.18 & 2.53 & 0.47 & 0.17 & 0.63 & 0.10 & 0.37 & 0.81 & 3.00 \\
\hline VO 0448 & $\begin{array}{l}\text { Tomato, raw (incl juice, } \\
\text { incl paste, excl canned) }\end{array}$ & 0.04 & 64.18 & 2.57 & 35.59 & 1.42 & 80.59 & 3.22 & 43.15 & 1.73 & 11.69 & 0.44 & 0.17 & 0.63 & 0.10 & 0.37 & 0.81 & 3.00 \\
\hline VO 0448 & $\begin{array}{l}\text { Tomato, raw (incl juice, } \\
\text { incl canned, excl paste) }\end{array}$ & 0.04 & 55.49 & 2.22 & 35.44 & 1.42 & 75.65 & 3.03 & 27.00 & 1.08 & 11.06 & 0.38 & 0.17 & 0.63 & 0.10 & 0.37 & 0.81 & 3.00 \\
\hline VO 0448 & $\begin{array}{l}\text { Tomato, raw (incl paste, } \\
\text { incl canned, excl juice) }\end{array}$ & 0.04 & 68.22 & 2.73 & 35.98 & 1.44 & 81.33 & 3.25 & 54.01 & 2.16 & 9.61 & 0.46 & 0.17 & 0.63 & 0.10 & 0.37 & 0.81 & 3.00 \\
\hline VO 0448 & $\begin{array}{l}\text { Tomato, raw (incl juice, } \\
\text { excl paste, excl canned) }\end{array}$ & 0.04 & 51.36 & 2.05 & 34.99 & 1.40 & 74.14 & 2.97 & 15.64 & 0.63 & 11.59 & 0.36 & 0.17 & 0.63 & 0.10 & 0.37 & 0.81 & 3.00 \\
\hline VO 0448 & $\begin{array}{l}\text { Tomato, raw (incl paste, } \\
\text { excl juice, excl canned) }\end{array}$ & 0.04 & 64.09 & 2.56 & 35.52 & 1.42 & 79.82 & 3.19 & 42.65 & 1.71 & 8.98 & 0.44 & 0.17 & 0.63 & 0.10 & 0.37 & 0.81 & 3.00 \\
\hline VO 0448 & $\begin{array}{l}\text { Tomato, raw (incl canned, } \\
\text { excl juice, excl paste) }\end{array}$ & 0.04 & 55.41 & 2.22 & 35.38 & 1.42 & 74.88 & 3.00 & 26.50 & 1.06 & 10.96 & 0.38 & 0.17 & 0.63 & 0.10 & 0.37 & 0.81 & 3.00 \\
\hline VO 0448 & Tomato, raw & 0.04 & 51.27 & 2.05 & 34.92 & 1.40 & 73.37 & 2.93 & 15.15 & 0.61 & 9.51 & 0.36 & 0.17 & 0.63 & 0.10 & 0.37 & 0.81 & 3.00 \\
\hline - & Tomato, canned (\& peeled) & 0.0016 & 2.66 & 0.00 & 0.30 & 0.00 & 0.97 & 0.00 & 7.31 & 0.01 & 8.88 & 0.00 & 0.17 & 0.63 & 0.10 & 0.37 & 0.81 & 3.00 \\
\hline - & $\begin{array}{l}\text { Tomato, paste (i.e. } \\
\text { concentrated tomato } \\
\text { sauce/puree) }\end{array}$ & 0.044 & 3.20 & 0.14 & 0.15 & 0.01 & 1.61 & 0.07 & 6.88 & 0.30 & 0.41 & 0.02 & 0.17 & 0.63 & 0.10 & 0.37 & 0.81 & 3.00 \\
\hline
\end{tabular}




\begin{tabular}{|c|c|c|c|c|c|c|c|c|c|c|c|c|c|c|c|c|c|c|}
\hline $\begin{array}{l}\text { Codex } \\
\text { Code }\end{array}$ & Commodity description & $\begin{array}{r}\text { STMR or } \\
\text { STMR-P } \\
\mathrm{mg} / \mathrm{kg}\end{array}$ & $\begin{array}{r}\text { G08 } \\
\text { (diet) }\end{array}$ & $\begin{array}{r}\text { G08 } \\
\text { intake }\end{array}$ & $\begin{array}{r}\text { G09 } \\
\text { (diet) }\end{array}$ & $\begin{array}{r}\text { G09 } \\
\text { intake }\end{array}$ & $\begin{array}{r}\text { G10 } \\
\text { (diet) }\end{array}$ & $\begin{array}{r}\text { G10 } \\
\text { intake }\end{array}$ & $\begin{array}{r}\text { G11 } \\
\text { (diet) }\end{array}$ & $\begin{array}{r}\text { G11 } \\
\text { intake }\end{array}$ & $\begin{array}{r}\text { G12 } \\
\text { (diet) }\end{array}$ & $\begin{array}{r}\text { G12 } \\
\text { intake }\end{array}$ & $\begin{array}{r}\text { G13 } \\
\text { (diet) }\end{array}$ & $\begin{array}{r}\text { G13 } \\
\text { intake }\end{array}$ & $\begin{array}{r}\text { G14 } \\
\text { (diet) }\end{array}$ & $\begin{array}{r}\text { G14 } \\
\text { intak } \\
\text { e }\end{array}$ & $\begin{array}{r}\text { G15 } \\
\text { (diet) }\end{array}$ & $\begin{array}{r}\text { G15 } \\
\text { intake }\end{array}$ \\
\hline JF 0448 & $\begin{array}{l}\text { Tomato, juice (single } \\
\text { strength, incl concentrated) }\end{array}$ & 0.006 & 0.10 & 0.00 & 0.10 & 0.00 & 0.61 & 0.00 & 0.40 & 0.00 & 0.52 & 0.00 & 0.17 & 0.63 & 0.10 & 0.37 & 0.81 & 3.00 \\
\hline VL 0502 & Spinach, raw & 3.7 & 1.76 & 6.51 & 13.38 & 49.51 & 2.94 & 10.88 & 5.53 & 20.46 & 0.10 & 0.37 & 0.17 & 0.63 & 0.10 & 0.37 & 0.81 & 3.00 \\
\hline VP 0064 & $\begin{array}{l}\text { Peas, green, without pods, } \\
\text { raw (i.e. immature seeds } \\
\text { only) (Pisum spp) }\end{array}$ & 0.09 & 1.99 & 0.18 & 2.72 & 0.24 & 4.26 & 0.38 & 4.23 & 0.38 & $\mathrm{NC}$ & - & 0.21 & 0.02 & 0.10 & 0.01 & 5.51 & 0.50 \\
\hline \multicolumn{3}{|c|}{ Total intake (ug/person) } & \multicolumn{2}{|r|}{259} & \multicolumn{2}{|r|}{91.6} & \multicolumn{2}{|r|}{162.2} & \multicolumn{2}{|r|}{255.1} & \multicolumn{2}{|r|}{33.3} & \multicolumn{2}{|r|}{9.6} & \multicolumn{2}{|r|}{7.4} & \multicolumn{2}{|r|}{262.5} \\
\hline \multicolumn{3}{|c|}{ Bodyweight per region $(\mathrm{kg} \mathrm{bw})=$} & \multicolumn{2}{|r|}{60} & \multicolumn{2}{|r|}{55} & \multicolumn{2}{|r|}{60} & \multicolumn{2}{|r|}{60} & \multicolumn{2}{|r|}{60} & \multicolumn{2}{|r|}{60} & \multicolumn{2}{|r|}{60} & \multicolumn{2}{|r|}{60} \\
\hline \multicolumn{3}{|r|}{$\% \mathrm{ADI}=$} & \multicolumn{2}{|r|}{$0.11 \%$} & \multicolumn{2}{|r|}{$0.04 \%$} & \multicolumn{2}{|r|}{$0.07 \%$} & \multicolumn{2}{|r|}{$0.11 \%$} & \multicolumn{2}{|r|}{$0.01 \%$} & & $0.004 \%$ & \multicolumn{2}{|c|}{$0.003 \%$} & \multicolumn{2}{|r|}{$0.109 \%$} \\
\hline
\end{tabular}




\begin{tabular}{|c|c|c|c|c|c|c|}
\hline Codex Code & Commodity description & $\begin{array}{r}\text { STMR or STMR-P } \\
(\mathbf{m g} / \mathbf{k g})\end{array}$ & G16 (diet) & G16 intake & G17 (diet) & G17 intake \\
\hline FB 2008 & Small fruit vine climbing, raw (incl processed) (i.e. grapes) & 0.21 & 0.74 & 0.16 & 44.23 & 9.29 \\
\hline FB 0269 & Grape, raw (incl must, incl dried, incl juice, incl wine ) & 0.21 & 0.74 & 0.16 & 44.23 & 9.29 \\
\hline FB 0269 & Grape, raw (incl must, incl dried, incl wine, excl juice) & 0.21 & 0.74 & 0.16 & 44.23 & 9.29 \\
\hline FB 0269 & Grape, raw (incl must, incl juice, incl wine, excl dried) & 0.21 & 0.73 & 0.15 & 44.12 & 9.27 \\
\hline FB 0269 & Grape, raw (incl dried, incl juice, incl wine, excl must) & 0.21 & 0.74 & 0.16 & 44.04 & 9.25 \\
\hline FB 0269 & Grape, raw (incl must, incl dried, incl juice, excl wine) & 0.21 & 0.10 & 0.02 & 0.40 & 0.08 \\
\hline FB 0269 & Grape, raw (incl must, incl dried, excl juice, excl wine) & 0.21 & 0.10 & 0.02 & 0.40 & 0.08 \\
\hline FB 0269 & Grape, raw (incl must, incl juice, excl dried, excl wine) & 0.21 & 0.10 & 0.02 & 0.28 & 0.06 \\
\hline FB 0269 & Grape, raw (incl must, incl wine, excl dried, excl juice) & 0.21 & 0.73 & 0.15 & 44.12 & 9.27 \\
\hline FB 0269 & Grape, raw (incl dried, incl juice, excl wine, excl must) & 0.21 & 0.10 & 0.02 & 0.21 & 0.04 \\
\hline FB 0269 & Grape, raw (incl dried, incl wine, excl must, excl juice) & 0.21 & 0.74 & 0.16 & 44.04 & 9.25 \\
\hline FB 0269 & Grape, raw (incl juice, incl wine, excl must, excl dried) & 0.21 & 0.73 & 0.15 & 43.92 & 9.22 \\
\hline FB 0269 & Grape, raw (incl must, excl dried, excl juice, excl wine) & 0.21 & 0.10 & 0.02 & 0.28 & 0.06 \\
\hline FB 0269 & Grape, raw (incl dried, excl must, excl juice, excl wine) & 0.21 & 0.10 & 0.02 & 0.21 & 0.04 \\
\hline FB 0269 & Grape, raw (incl juice, excl must, excl dried, excl wine) & 0.21 & 0.10 & 0.02 & 0.10 & 0.02 \\
\hline FB 0269 & Grape, raw (incl wine, excl must, excl dried, excl juice) & 0.21 & 0.73 & 0.15 & 43.92 & 9.22 \\
\hline FB 0269 & Grape, raw & 0.21 & 0.10 & 0.02 & 0.10 & 0.02 \\
\hline - & Grape must & 0.13 & 0.10 & 0.01 & 0.19 & 0.02 \\
\hline DF 0269 & Grape, dried (= currants, raisins and sultanas) & 0.29 & 0.10 & 0.03 & 0.10 & 0.03 \\
\hline
\end{tabular}




\begin{tabular}{|c|c|c|c|c|c|c|}
\hline Codex Code & Commodity description & $\begin{array}{l}\text { STMR or STMR-P } \\
\qquad(\mathrm{mg} / \mathrm{kg})\end{array}$ & G16 (diet) & G16 intake & G17 (diet) & G17 intake \\
\hline JF 0269 & Grape juice & 0.034 & 0.10 & 0.00 & $\mathrm{NC}$ & - \\
\hline - & Grape wine (incl vermouths) & 0.029 & 0.52 & 0.02 & 31.91 & 0.93 \\
\hline - & Onions, mature bulbs, dry & 0.01 & 9.61 & 0.10 & 2.11 & 0.02 \\
\hline - & Onions, green, raw & 0.6 & $\mathrm{NC}$ & - & 6.30 & 3.78 \\
\hline VB 0041 & Cabbages, head, raw & 0.14 & 0.10 & 0.01 & $\mathrm{NC}$ & - \\
\hline VB 0400 & Broccoli, raw & 0.22 & 0.10 & 0.02 & $\mathrm{NC}$ & - \\
\hline VB 0404 & Cauliflower, raw & 0.08 & 0.10 & 0.01 & $\mathrm{NC}$ & - \\
\hline VC 0046 & Melons, raw (excl watermelons) & 0.03 & 0.10 & 0.00 & $\mathrm{NC}$ & - \\
\hline VC 0424 & Cucumber, raw & 0.06 & 0.10 & 0.01 & 0.10 & 0.01 \\
\hline VO 0448 & Tomato, raw (incl juice, incl paste, incl canned) & 0.04 & 0.10 & 0.04 & $\mathrm{NC}$ & - \\
\hline VO 0448 & Tomato, raw (incl juice, incl paste, excl canned) & 0.04 & 2.00 & 0.08 & 12.50 & 0.50 \\
\hline VO 0448 & Tomato, raw (incl juice, incl canned, excl paste) & 0.04 & 1.88 & 0.08 & 12.50 & 0.50 \\
\hline VO 0448 & Tomato, raw (incl paste, incl canned, excl juice) & 0.04 & 1.04 & 0.04 & 0.11 & 0.00 \\
\hline VO 0448 & Tomato, raw (incl juice, excl paste, excl canned) & 0.04 & 1.99 & 0.08 & 12.48 & 0.50 \\
\hline VO 0448 & Tomato, raw (incl paste, excl juice, excl canned) & 0.04 & 0.92 & 0.04 & 0.11 & 0.00 \\
\hline VO 0448 & Tomato, raw (incl canned, excl juice, excl paste) & 0.04 & 1.88 & 0.08 & 12.48 & 0.50 \\
\hline VO 0448 & Tomato, raw & 0.04 & 1.04 & 0.04 & 0.10 & 0.00 \\
\hline - & Tomato, canned (\& peeled) & 0.0016 & 0.92 & 0.04 & 0.10 & 0.00 \\
\hline - & Tomato, paste (i.e. concentrated tomato sauce/puree) & 0.044 & 0.10 & 0.00 & $\mathrm{NC}$ & - \\
\hline JF 0448 & Tomato, juice (single strength, incl concentrated) & 0.006 & 0.24 & 0.01 & 3.10 & 0.14 \\
\hline VL 0502 & Spinach, raw & 3.7 & 0.10 & 0.00 & 0.10 & 0.00 \\
\hline VP 0064 & $\begin{array}{l}\text { Peas, green, without pods, raw (i.e. immature seeds only) } \\
\text { (Pisum spp) }\end{array}$ & 0.09 & $\mathrm{NC}$ & - & $\mathrm{NC}$ & - \\
\hline
\end{tabular}




\begin{tabular}{|c|c|c|c|c|c|c|}
\hline Codex Code & Commodity description & $\begin{array}{c}\text { STMR or STMR-P } \\
(\mathrm{mg} / \mathrm{kg})\end{array}$ & G16 (diet) & G16 intake & G17 (diet) & G17 intake \\
\hline \multicolumn{3}{|r|}{ Total intake (ug/person) } & \multicolumn{2}{|c|}{2.7} & \multicolumn{2}{|r|}{90.7} \\
\hline \multicolumn{3}{|c|}{ Bodyweight per region $(\mathrm{kg} \mathrm{bw})=$} & & 0 & \multicolumn{2}{|r|}{60} \\
\hline \multicolumn{3}{|r|}{$\% \mathrm{ADI}=$} & & \multicolumn{3}{|r|}{$0.038 \%$} \\
\hline
\end{tabular}

